\title{
The IFNL3/4 $\Delta G$ variant increases susceptibility to cytomegalovirus retinitis among HIV-infected patients
}

\author{
Stéphanie Bibert ${ }^{\mathrm{a}}$, Agnieszka Wojtowicz ${ }^{\mathrm{a}}$, Patrick Tafféb, \\ Oriol Manuel ${ }^{\mathrm{a}}$, Enos Bernasconic ${ }^{\mathrm{c}}$, Hansjakob Furrer ${ }^{\mathrm{d}}$, \\ Huldrych F. Günthard ${ }^{\mathrm{e}}$, Matthias Hoffmann ${ }^{\mathrm{f}}$, Laurent Kaiserg, \\ Michael Osthoff ${ }^{\mathrm{h}}$, Matthias Cavassini ${ }^{\mathrm{a}, *}$, Pierre-Yves Bochud ${ }^{\mathrm{a}, *}$, \\ the Swiss HIV Cohort Study ${ }^{\dagger}$
}

\begin{abstract}
Background: Cytomegalovirus (CMV) retinitis is a major cause of visual impairment and blindness among patients with uncontrolled HIV infections. Whereas polymorphisms in interferon-lambda 3 (IFNL3, previously named IL28B) strongly influence the clinical course of hepatitis $C$, few studies examined the role of such polymorphisms in infections due to viruses other than hepatitis $C$ virus.

Objectives: To analyze the association of newly identified IFNL3/4 variant rs368234815 with susceptibility to CMV-associated retinitis in a cohort of HIV-infected patients.

Design and methods: This retrospective longitudinal study included 4884 white patients from the Swiss HIV Cohort Study, among whom 1134 were at risk to develop CMV retinitis $\left(\mathrm{CD}^{+}\right.$nadir $<100 / \mu \mathrm{l}$ and positive CMV serology). The association of CMV-associated retinitis with rs368234815 was assessed by cumulative incidence curves and multivariate Cox regression models, using the estimated date of HIV infection as a starting point, with censoring at death and/or lost follow-up.

Results: A total of 40 individuals among 1134 patients at risk developed CMV retinitis. The minor allele of $r s 368234815$ was associated with a higher risk of CMV retinitis (logrank test $P=0.007$, recessive mode of inheritance). The association was still significant in a multivariate Cox regression model (hazard ratio $2.31,95 \%$ confidence interval 1.09-4.92, $P=0.03$ ), after adjustment for $\mathrm{CD}^{+}{ }^{+}$nadir and slope, HAART and HIV-risk groups.
\end{abstract}

\footnotetext{
anfectious Diseases Service, Department of Medicine, University Hospital and University of Lausanne, ${ }^{\mathrm{b}}$ Institute for Social and Preventive Medicine, University (IUMSP), University Hospital of Lausanne, Lausanne, 'Division of Infectious diseases, Regional hospital of Lugano, Lugano, ${ }^{\mathrm{d} D e p a r t m e n t ~ o f ~ I n f e c t i o u s ~ D i s e a s e s, ~ B e r n ~ U n i v e r s i t y ~ H o s p i t a l ~ a n d ~ U n i v e r s i t y ~ o f ~ B e r n, ~ B e r n, ~}{ }^{\mathrm{e} D i v i s i o n}$ of Infectious Diseases and Hospital Epidemiology, University Hospital Zurich, University of Zurich, Zurich, ${ }^{\mathrm{f}}$ Division of Infectious Diseases and Hospital Epidemiology, Department of Internal Medicine, Cantonal Hospital St Gallen, St Gallen, ${ }^{\mathrm{B}}$ Laboratory of Virology, Division of Infectious Diseases and Division of Laboratory Medicine, University Hospital of Geneva and Medical School, University of Geneva, Geneva, and hivision of Infectious Diseases and Hospital Epidemiology, University Hospital Basel, Basel, Switzerland.
}

Correspondence to Pierre-Yves Bochud, MD, Infectious Diseases Service, CHUV, Rue du Bugnon 46, 1011 Lausanne, Switzerland.

Tel: +41 2131443 79; fax: +41 2131440 60; e-mail: Pierre-Yves.Bochud@chuv.ch

* Matthias Cavassini and Pierre-Yves Bochud contributed equally to the manuscript.

${ }^{\dagger}$ Members of the Swiss HIV Cohort Study group are listed in the acknowledgement section.

Received: 1 May 2014; revised: 5 June 2014; accepted: 5 June 2014. 
Conclusion: We reported for the first time an association between an IFNL3/4 polymorphism and susceptibility to AIDS-related CMV retinitis. IFNL3/4 may influence immunity against viruses other than HCV. ๑ 2014 Wolters Kluwer Health | Lippincott Williams \& Wilkins

AIDS 2014, 28:1885-1889

\section{Keywords: AIDS, cytomegalovirus retinitis, HIV, IFNL3, immunogenetics, polymorphism}

\section{Introduction}

Cytomegalovirus (CMV) retinitis is a major cause of visual impairment and blindness among patients with uncontrolled HIV infections, insufficient drug compliance or therapy intolerance [1]. The disease is characterized by progressive necrosis that can lead to retinal detachment, optic atrophy and finally loss of vision [2-4].

Although individuals can remain asymptomatic for extended periods of time with low $\mathrm{CD}^{+}$cell counts, others rapidly develop retinitis when their $\mathrm{CD}^{+}$cell count drops below 50-100 $\mathrm{CD}^{+}$cells/ $\mu$ l. Such differences in the onset and/or susceptibility of the disease may be partly due to host genetic factors. Among African Americans, a promoter haplotype within C-C chemokine receptor type 5 (CCR 5) and a polymorphism in the stromal cell-derived factor 1 (SDF1) were associated with increased risk of retinitis progression $[5,6]$. In white patients, different haplotypes of the interleukin (IL)-10 receptor subunit 1 (IL10-R1) [5] and polymorphisms within tumor necrosis factor (TNF) [7] were linked to susceptibility to CMV retinitis.

Interferon-lambda 3 (IFNL3) (previously designated as IL28B) belongs to the type III IFN family and was reported as a major determinant of hepatitis $\mathrm{C}$ virus (HCV) clearance. A TT/-G substitution (rs368234815) introducing a frameshift in the DNA sequence was shown to be the best marker of HCV clearance [8,9]. The minor $-G$ allele was recently associated with the expression of a novel IFN-like protein called IFNL4 [8]. We showed that this allele was also associated with reduced IFNL3 expression $[8,9]$. So far, only a few studies examined whether IFNL3/4 polymorphisms influence immune responses against viruses other than HCV [10-12]. We examined the role of this polymorphism in the development of CMV retinitis among patients from the Swiss HIV Cohort Study (SHCS).

\section{Material and methods}

\section{Study patients}

Patients were included from the Swiss HIV Cohort Study, an ongoing multicenter prospective study of HIVinfected patients enrolled at seven major Swiss hospitals and their local affiliated centers since 1988. White patients with available samples for DNA extraction, written consent for genetic studies and a CD4 ${ }^{+}$nadir below $400 / \mu l$ were selected. Demographic characteristics including age, duration of HIV infection, sex, CD4 ${ }^{+}$T-cell count nadir, other opportunistic infections, HIV maximal viral load and HAART use were extracted from clinical databases. Patients at risk to develop CMV retinitis were defined as those with a positive CMV serology and a $\mathrm{CD} 4^{+}$T-cell nadir below $100 / \mu 1$. CMV retinitis was defined by characteristic ophthalmoscopic findings (discrete patches of retinal whitening with distinct borders speeding in a centrifugal manner, frequently associated with retinal vasculitis, hemorrhage and necrosis) followed by evidence of response to CMV treatment (residual retinal scarring and atrophy with retinal pigment epithelial mottling). The disease was considered presumptive unless proven by a positive CMV PCR detection.

\section{Single-nucleotide polymorphism genotyping}

Genomic DNAs isolated either from blood or cell pellets were genotyped using a customized Golden Gate Genotyping Assay on Veracode platform (Illumina, San Diego, California, USA) or a Competitive Allele Specific PCR (KASP) system (LGC Genomics, former KBioscience, Hoddesdon, Herts, UK; http://www.lgcgenomics.com/ kaspchallenge).

\section{Statistical analysis}

Statistical analyses were performed using Stata (version 13.1; StataCorp LP, College Station, Texas, USA). Hardy-Weinberg equilibrium test was assessed using the program genhw, implemented in Stata. The association of IFNL3/4 polymorphism with CMV retinitis for the additive, dominant and recessive modes of inheritance was assessed by cumulative incidence curves (i.e. 1 minus Kaplan-Meier to estimate the cumulative probability of retinitis), using the estimated date of HIV infection as a starting point, with censoring at death and/or lost follow-up. Proportional hazard assumption was verified by using the stphtest command implemented in Stata. Estimated dates of HIV infection and $\mathrm{CD} 4^{+}$slopes (rate of $\mathrm{CD} 4^{+}$depletion in the absence of HAART) in both incident and prevalent cases were obtained by using a joint back calculation model, as described previously [13]. The $\mathrm{CD} 4^{+}$nadir square root was used to obtain a normally distributed variable. The association between CMV retinitis and genetic variables 
was first assessed using the log-rank test. Demographic and clinical factors that were potentially associated with CMV retinitis were then analyzed in univariate and multivariate Cox regression models. Stepwise (i.e. backward) regression $(P<0.2)$ was used to determine which variables were independently associated with the endpoint.

\section{Results}

The study included 4884 white patients with consent for host genetic studies from the SHCS, among whom 1134 were at risk of developing CMV retinitis $\left(\mathrm{CD} 4^{+}\right.$nadir $<100 / \mu 1$ and positive CMV serology; Supplementary Table 1, http://links.lww.com/QAD/A544). In this high-risk group, most patients were male $(78 \%)$ and the most frequent risk factors for HIV infection included homosexual contact (43\%), heterosexual contact (32\%) and drug use (21\%). The median age at estimated time of HIV infection was 31 years [interquartile range (IQR) 14]. The median $\mathrm{CD} 4^{+}$T-cell count nadir and $\mathrm{CD} 4^{+}$ slopes were 39.5/ $\mu \mathrm{l}$ (IQR 47) and -2.66 (IQR 0.97), respectively. CMV retinitis was diagnosed in $40 / 1134$ individuals ( 29 proven and 11 presumptive).

Patients who carried two copies of the minor $-\mathrm{G}$ allele of IFNL3/4 rs368234815 polymorphisms had a significantly higher cumulative incidence of CMV retinitis (logrank test $P=0.007$; Fig. 1). The association was still significant in a multivariate Cox regression model [hazard ratio 2.31 , 95\% confidence interval (CI) 1.09-4.92,
$P=0.03]$. Covariates that remained in the model after stepwise (backward) selection were the $\mathrm{CD}^{+}{ }^{+}$cell count nadir square root (hazard ratio 0.73 , 95\% CI $0.64-0.84$, $P<0.001$ ), the $\mathrm{CD}^{+}$slope (hazard ratio $0.73,95 \% \mathrm{CI}$ $0.49-1.07, P=0.11$ ), the use of HAART (hazard ratio $0.40,95 \%$ CI $0.18-0.88, P=0.02)$, as well as the HIV transmission mode (heterosexual contact, hazard ratio $0.51,95 \%$ CI $0.23-1.09, P=0.08$; intravenous (i.v.) drug use, hazard ratio $0.35,95 \% \mathrm{CI} 0.13-0.93, P=0.04$, using MSM as reference; Table 1 and Supplementary Table 2, http://links.lww.com/QAD/A544).

\section{Discussion}

Polymorphisms in IFNL3/4 have a dramatic influence on HCV clearance, either spontaneously or in response to IFN-base therapy $[8,9]$. Few studies examined whether these polymorphisms influence susceptibility to other viral pathogens. In this study, we report an association between the newly identified IFNL3/4 mutant rs368234815 allele and susceptibility to CMV retinitis among HIV-infected patients.

The association between the $r s 368234815$ polymorphism and susceptibility to CMV retinitis is consistent with studies performed in the field of hepatitis $\mathrm{C}$, as the same allele was associated with reduced HCV clearance, either spontaneously or after IFN-based treatment. In addition, we recently showed that this polymorphism is associated with increased CMV infection in a cohort of 1021 solid organ transplant recipients [14]. This suggests that IFNLs

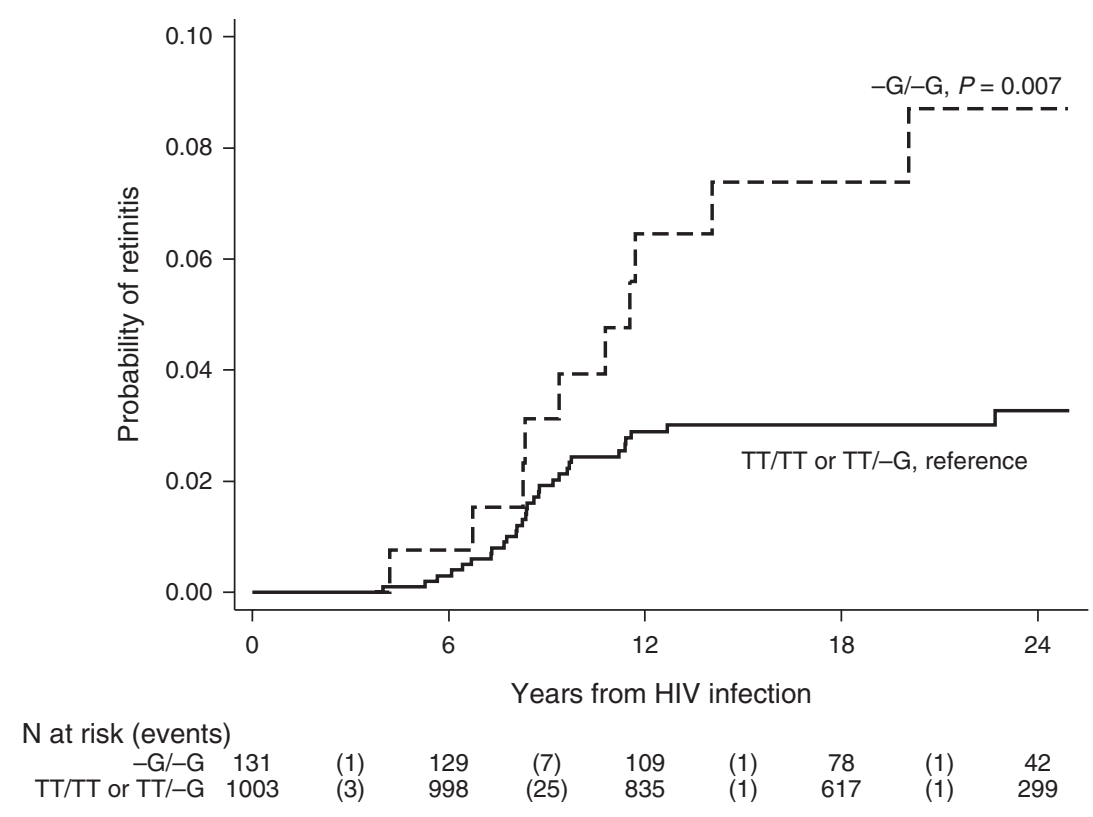

Fig. 1. Cumulative incidence curves (i.e. 1 minus Kaplan-Meier-estimated probability) of cytomegalovirus retinitis according to IFNL3/4 rs368234815 polymorphism. The estimated date of HIV infection was used as a starting point, with censoring at death or lost follow-up. 
Table 1. Independent risk factors associated with cytomegalovirus retinitis.

\begin{tabular}{|c|c|c|c|c|}
\hline \multirow[b]{2}{*}{ Variable } & \multicolumn{2}{|c|}{ Univariate } & \multicolumn{2}{|c|}{ Multivariate } \\
\hline & $\mathrm{HR}(95 \% \mathrm{Cl})^{\mathrm{a}}$ & $P$ & $\mathrm{HR}(95 \% \mathrm{Cl})^{\mathrm{b}}$ & $P$ \\
\hline Sex & $0.72(0.32-1.64)$ & 0.44 & & \\
\hline Age at estimated date of infection (continuous) & $1.00(0.97-1.03)$ & 0.99 & & \\
\hline $\mathrm{CD}^{+}$nadir (sqrt transformed, continuous) & $0.71(0.63-0.81)$ & $<0.001$ & $0.73(0.64-0.84)$ & $<0.001$ \\
\hline $\mathrm{CD}^{+}$slope (continuous) & $0.64(0.45-0.92)$ & 0.01 & $0.73(0.49-1.07)$ & 0.11 \\
\hline Maximal HIV RNA load (continuous) & $1.38(0.87-2.17)$ & 0.17 & & \\
\hline HAART (time-dependent covariate) ${ }^{\mathrm{a}}$ & $0.48(0.24-0.97)$ & 0.04 & $0.40(0.18-0.88)$ & 0.02 \\
\hline \multicolumn{5}{|l|}{ Risk factors } \\
\hline MSM & Ref. & & & \\
\hline Hetero & $0.57(0.27-1.20)$ & 0.14 & $0.51(0.23-1.09)$ & 0.08 \\
\hline Drug user & $0.40(0.15-1.06)$ & 0.06 & $0.35(0.13-0.93)$ & 0.04 \\
\hline Other & $0.53(0.07-3.93)$ & 0.54 & & \\
\hline HCV coinfection & $1.03(0.55-1.93)$ & 0.92 & & \\
\hline Active HBV infection & $0.88(0.21-3.63)$ & 0.86 & & \\
\hline IFNL3 rs368234815 (-G/-G versus TT/TT or TT/-G) & $2.59(1.26-5.29)$ & 0.009 & $2.31(1.09-4.92)$ & 0.03 \\
\hline
\end{tabular}

Stepwise (i.e. backward) regression $(P<0.2)$ was used to determine which variables were independently associated with the endpoint. $\mathrm{Cl}$, confidence interval; $\mathrm{HBV}$, hepatitis $B$ virus; $\mathrm{HCV}$, hepatitis $\mathrm{C}$ virus; $\mathrm{HR}$, hazard ratio.

${ }^{a}$ The proportional hazard assumption was verified for all variables with the exception of HAART $(P=0.03$ in the univariate model, $P=0.04$ in the multivariate model). However, the association between IFNL3 rs368234815 and CMV retinitis was similar when HAART was removed from the multivariate model (HR 2.53,95\% Cl 1.20-5.35, $P=0.015$ ) (see supplementary Table 2, http://links.lww.com/QAD/A544).

${ }^{\mathrm{b}}$ The association between $r s 368234815$ and CMV retinitis was still significant when anti-CMV treatment was added into the model (HR $2.55,95 \%$ Cl 1.20-5.43, $P=0.015)$.

may be involved in the immune response to an extended range of viral pathogens.

Whereas the functional relevance of this association remains to be elucidated, increasing evidences suggest a role for IFNLs in the immune response against herpesviruses, including CMV. Immune cells can detect CMV by using two main families of pattern recognition receptors - the toll-like receptors (TLR2, TLR3, TLR7, TLR8, TLR9) and the RIG-like receptors (Melanoma Differentiation-Associated protein 5 (MDA5) and retinoic acid-inducible gene 1 (RIG-I)), which lead to the production of IFNs. IFNs promote the induction of IFNstimulated genes (ISGs) which were shown to exert antiviral activities against a broad range of viruses. For instance, colonic cells from mice infected with murine CMV were shown to produce increased amounts of IFNLs [15]. In turn, IFNLs up-regulated STAT1 phosphorylation and ISG production in different human intestinal epithelial cells which results in an increased IFN response due to the expression of ISGs [15]. Recent studies using different models of CMV retinitis highlighted the role of IFNs in the development of Th1 immune responses $[16,17]$ and the contribution of IFNL3 in the regulation of T-cell function and natural killer (NK)-cell cytotoxicity [18].

The rs 368234815 polymorphism was shown to influence both IFNL3 and IFN- $\boldsymbol{\gamma}$-inducible protein 10 (IP-10) mRNA expression in peripheral blood mononuclear cells (PBMCs) stimulated with Poly(I:C) and to promote the expression of IFNL4 $[8,9]$. Thus, increased susceptibility to retinitis in individuals carrying the polymorphisms may result from insufficient IFNL3 and/or the production of
IFNL4. Yet, the mechanisms by which IFNL4 (a cytokine predicted to exert antiviral activities) can be associated with increased susceptibility to viral infections has still not been elucidated [19].

Like most genetic association studies, our study has several limitations. Whereas the diagnosis of CMV retinitis is based on stringent clinical and laboratory criteria, the diagnosis of CMV disease is ill-defined, and its association with IFNL3/4 polymorphisms was not analyzed in this study. Whereas most cases of CMV retinitis were diagnosed as proven (PCR-documented), a few cases were classified as presumptive (based on a positive CMV serology and a typical clinical presentation diagnosed by an ophthalmologist). Finally, the number of CMV retinitis patients carrying the risk genotype was relatively small. Further studies are needed to replicate the association reported in this study, and additional work is needed to understand the biological role of IFLNs in CMV retinitis.

\section{Acknowledgements}

The authors thank all patients from the SHCS, as well as collaborators from the clinical, laboratory and data centers and all study nurses.

The project was supported by SHCS project No 613. The SHCS is supported by the Swiss National Foundation (SNF grant No 33CS30_148522) and by the SHCS research foundation. 
Author's contributions: S.B. performed sample management, DNA extraction, candidate SNP genotyping and wrote the manuscript.

A.W. performed SNP genotyping for the SHCS patients and data management.

P.T. contributed to statistical analyses.

M.C. and O.M. contributed to study design and manuscript writing.

Members of the SHCS group including E.B., H.F., H.F.G., M.H., L.K., M.O., and M.C. were directly involved in the follow-up of SHCS patients and case report forms management.

P.Y.B. designed the SHCS genetic project, obtained funding, supervised genotyping, performed data management and statistical analysis and wrote the manuscript.

All authors critically revised the manuscript.

The members of the Swiss HIV Cohort Study are: J. Barth, M. Battegay, E. Bernasconi, J. Böni, H.C. Bucher, C. Burton-Jeangros, A. Calmy, M. Cavassini, C. Cellerai, M. Egger, L. Elzi, J. Fehr, J. Fellay, M. Flepp, P. Francioli, H. Furrer, C.A. Fux, M. Gorgievski, H. Günthard, D. Haerry, B. Hasse, H.H. Hirsch, B. Hirschel, I. Hösli, C. Kahlert, L. Kaiser, O. Keiser, C. Kind, T. Klimkait, H. Kovari, B. Ledergerber, G. Martinetti, B. Martinez de Tejada, K. Metzner, N. Müller, D. Nadal, G. Pantaleo, A. Rauch, S. Regenass, M. Rickenbach, C. Rudin, P. Schmid, D. Schultze, F. Schöni-Affolter, J. Schüpbach, R. Speck, A. Telenti, A. Trkola, P. Vernazza, R. Weber, S. Yerly.

\section{Conflicts of interest}

P.Y.B. is supported by the Swiss National Foundation (320030_144054), the Leenaards Foundation, the SantosSuarez Foundation and the Loterie Romande. P.Y.B. is recipient of a Mérieux research grant.

\section{References}

1. Heiden D, Ford N, Wilson D, Rodriguez WR, Margolis T, Janssens $B$, et al. Cytomegalovirus retinitis: the neglected disease of the AIDS pandemic. PLoS Med 2007; 4:e334.

2. Jabs DA. Ocular manifestations of HIV infection. Trans Am Ophthalmol Soc 1995; 93:623-683.
3. Jabs DA, Van Natta ML, Holbrook JT, Kempen JH, Meinert CL, Davis MD, et al. Longitudinal study of the ocular complications of AIDS: 1. Ocular diagnoses at enrollment. Ophthalmology 2007; 114:780-786.

4. Jacobson MA, Stanley $\mathrm{H}$, Holtzer C, Margolis TP, Cunningham ET. Natural history and outcome of new AIDS-related cytomegalovirus retinitis diagnosed in the era of highly active antiretroviral therapy. Clin Infect Dis 2000; 30:231-233.

5. Sezgin E, Jabs DA, Hendrickson SL, Van Natta M, Zdanov A, Lewis RA, et al. Effect of host genetics on the development of cytomegalovirus retinitis in patients with AIDS. I Infect Dis 2010; 202:606-613.

6. Sezgin E, van Natta ML, Ahuja A, Lyon A, Srivastava S, Troyer JL, et al. Association of host genetic risk factors with the course of cytomegalovirus retinitis in patients infected with human immunodeficiency virus. Am J Ophthalmol 2011; 151:999$1006 \mathrm{e} 1004$.

7. Deghaide NH, Rodrigues Mde L, Castelli EC, Mendes-Junior CT, Figueiredo JF, Donadi EA. Tumor necrosis factor region polymorphisms are associated with AIDS and with cytomegalovirus retinitis. AIDS 2009; 23:1641-1647.

8. Prokunina-Olsson L, Muchmore B, Tang W, Pfeiffer RM, Park H, Dickensheets $\mathrm{H}$, et al. A variant upstream of IFNL3 (IL28B) creating a new interferon gene IFNL4 is associated with impaired clearance of hepatitis C virus. Nat Genet 2013; 45:164171.

9. Bibert S, Roger T, Calandra T, Bochud M, Cerny A, Semmo N, et al. IL28B expression depends on a novel TT/-G polymorphism which improves HCV clearance prediction. J Exp Med 2013; 210:1109-1116.

10. Ank N, West H, Bartholdy C, Eriksson K, Thomsen AR, Paludan SR. Lambda interferon (IFN-lambda), a type III IFN, is induced by viruses and IFNs and displays potent antiviral activity against select virus infections in vivo. J Virol 2006; 80:45014509.

11. Ank N, Iversen MB, Bartholdy C, Staeheli P, Hartmann R, Jensen $U B$, et al. An important role for type III interferon (IFNlambda/IL-28) in TLR-induced antiviral activity. I Immunol 2008; 180:2474-2485.

12. Schneider K, Meyer-Koenig U, Hufert FT. Human cytomegalovirus impairs the function of plasmacytoid dendritic cells in lymphoid organs. PLOS One 2008; 3:e3482.

13. Taffe $\mathrm{P}$, May M, Swiss HIVCS. A joint back calculation model for the imputation of the date of HIV infection in a prevalent cohort. Stat Med 2008; 27:4835-4853.

14. Manuel O, Wojtowicz A, Bibert S, Kralidis G, Egli A, Mueller N, et al. IL28B Polymorphisms influence cytomegalovirus viremia in solid organ transplant recipients. American Transplant Congress, Seattle May 18-22, 2013.

15. Brand S, Beigel F, Olszak T, Zitzmann K, Eichhorst ST, Otte JM, et al. IL-28A and IL-29 mediate antiproliferative and antiviral signals in intestinal epithelial cells and murine $\mathrm{CMV}$ infection increases colonic IL-28A expression. Am J Physiol Gastrointest Liver Physiol 2005; 289:G960-968.

16. Takase H, Futagami $Y$, Yoshida T, Kamoi K, Sugita S, Imai $Y$, et al. Cytokine profile in aqueous humor and sera of patients with infectious or noninfectious uveitis. Invest Ophthalmol Vis Sci 2006; 47:1557-1561.

17. Iyer JV, Connolly J, Agrawal R, Yeo TK, Lee B, Au B, et al. Cytokine analysis of aqueous humor in HIV patients with cytomegalovirus retinitis. Cytokine 2013; 64:541-547.

18. Bigger JE, Thomas CA 3rd, Atherton SS. NK cell modulation of murine cytomegalovirus retinitis. I Immunol 1998; 160:58265831.

19. Hamming OJ, Terczynska-Dyla E, Vieyres G, Dijkman $R$, Jorgensen $\mathrm{SE}$, Akhtar $\mathrm{H}$, et al. Interferon lambda 4 signals via the IFNlambda receptor to regulate antiviral activity against HCV and coronaviruses. EMBO / 2013; 32:3055-3065. 